\title{
HIGHLIGHTS
}

CELIAC DISEASE

\section{Celiac disease in children is associated with cesarean delivery}

A new study has found that cesarean delivery has a significant but moderate association with celiac disease in children.

Decker $e t$ al. conducted a retrospective, multicenter, case-control study to investigate a possible association between cesarean delivery and enteric inflammatory diseases.

"There were two reasons to do the work," explains Mathias Hornef, corresponding author of the study. "One is our previous work on postnatal acquisition of epithelial innate immune tolerance after birth in mice. [We] showed that the intestinal epithelium is tolerized subsequent to the exposure to environmental lipopolysaccharides that occurs during vaginal delivery. This tolerization was not observed in [cesarean] born mice." In addition, other studies have shown that the bacterial colonization of the enteric mucosal surface differs in children born by vaginal delivery versus cesarean delivery.

1,950 children and young adolescents were included in the present study. Data were collected from patients visiting a pediatric gastrointestinal outpatient clinic; patients visiting opthalmologic, orthodontic or dental outpatient clinics were used as controls.

The authors found significantly higher cesarean delivery rates among patients with celiac disease compared with controls (odds ratio 1.80 [95\% CI 1.13-2.88, $P=0.014])$. No association was found between IBD and cesarean delivery.

"The epidemiological data now need to be confirmed in additional larger studies," concludes Hornef. "Also, I think we now need to go back [to] the mouse

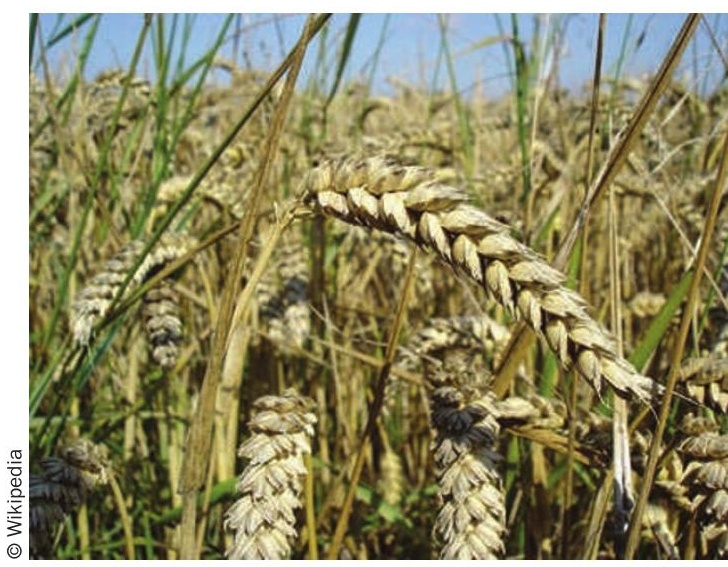

model and further delineate the functional consequences of ... enteric epithelial homeostasis, and the influence of the flora and environmental innate immune stimuli on the mucosal tissue."

Isobel Franks

Original article Decker, E. et al. Cesarean delivery is associated with celiac disease but not inflammatory bowel disease in children. Pediatrics 6, e1433-e1440 (2010) 